九州大学学術情報リポジトリ

Kyushu University Institutional Repository

\title{
Cont inuum damage mechanics modeling of composite laminates including transverse cracks
}

Okabe, Tomonaga

Department of Aerospace Engineering, Tohoku University

Onodera, Sota

Department of Aerospace Engineering, Tohoku University

Kumagai, Yuta

Department of Aerospace Engineering, Tohoku University

Nagumo, Yoshiko

Department of Aerospace Engineering, Tohoku University

http://hdl. hand le. net/2324/4403328

出版情報: International Journal of Damage Mechanics. 27 (6)，pp.877-895，2017-06-05. SAGE Publications

バージョン:

権利関係: (C) The authors. 


\title{
Continuum damage mechanics modeling of composite laminates including transverse cracks
}

\author{
Tomonaga Okabe ${ }^{1}$, Sota Onodera ${ }^{1}$, Yuta Kumagai ${ }^{1}$ and Yoshiko Nagumo ${ }^{1}$ \\ ${ }^{1}$ Department of Aerospace Engineering, Tohoku University
}

\section{Corresponding author:}

Tomonaga Okabe, Department of Aerospace Engineering, Tohoku University, 6-6-01, Aramaki-Aza-Aoba, Aoba-ku, Sendai, 980-8579, JAPAN

Email: okabe@plum.mech.tohoku.ac.jp

\begin{abstract}
In this study, the continuum damage mechanics model for predicting the stiffness reduction of composite laminates including transverse cracks is formulated as a function of crack density. To formulate the model, first the damage variable in the direction normal to the fiber of a ply including transverse cracks is derived. The damage variable is derived by the model assuming a plane strain field in the isotropic plane and
\end{abstract}


using the Gudmundson-Zang model for comparison. The effective compliance based on the strain equivalent principle proposed by Murakami and his colleagues and classical laminate theory are then used to formulate the elastic moduli of laminates of arbitrary lay-up configurations as a function of the damage variable. Finally, the results obtained from this model are compared to the finite element analysis (FEA) reported in previous studies. The model proposed in this paper can predict the stiffness of laminates containing damage due to transverse cracks (or surface crack) from just the mechanical properties of a ply and the lay-up configurations. Furthermore, this model can precisely predict the FEA results and experiment results for the elastic moduli of the laminate of arbitrary lay-up configuration, such as cross-ply, angle ply, and quasi-isotropic, including transverse cracks. This model only considers the damage of the transverse crack; it does not consider damage such as delamination. However, this model seems to be effective in the early stage of damage formation when transverse cracking mainly occurs. The model assuming plane strain field in the isotropic plane which is proposed in this paper can calculate the local stress distribution in a ply including transverse cracks as a function of crack density. The damage evolution of transverse cracks can thus be simulated by determining the fracture criterion. 


\section{Keywords}

Damage, transverse, crack, analysis, laminates

\section{Introduction}

Recently, composite materials such as fiber-reinforced plastic (FRP) have been used as a main component in primary structures. Generally, laminates made by stacking unidirectional fiber-reinforced lamina, which have predefined mechanical properties, are commonly used because unidirectional fiber-reinforced plastic is highly anisotropic. There are some kinds of laminate, such as cross-ply laminate, which has fiber angles of $0^{\circ}$ and $90^{\circ}$ to the loading direction, angle-ply laminate, which has fiber angles of $\pm \theta^{\circ}$ to the loading direction, and quasi-isotropic laminate, which has isotropic elastic moduli in the plane. To utilize these laminates of arbitrary lay-up configurations, it is essential to know the fracture process of the laminate. Fiber-reinforced laminate exhibits specific fracture processes such as transverse cracks (cracks in a direction parallel to the fiber), delamination, and breakage of the fiber. In these fracture processes, transverse cracking occurs in the earliest stage. The initiation of transverse cracking causes stress 
redistribution, resulting in delamination and breakage of the fiber due to the stress concentration at the crack tip. Therefore, it is important to clarify the mechanical behavior of laminate, including transverse cracking.

There have been various studies on laminate including transverse cracks. ${ }^{1-22)}$ The simplest method considering the effect of transverse cracking is called the ply discount method. ${ }^{1,2)}$ In this method, the stiffness of the ply including transverse cracks is ignored, resulting in a lower estimation of the stiffness of the laminate. Moreover, it is impossible to estimate the decrease in stiffness caused by changing the transverse crack density using this method. Therefore, continuum damage mechanics is a valid approach to this issue. In continuum damage mechanics, the internal state variable and damage variable are utilized to consider the effect of transverse cracks on the reduction of stiffness shown in previous papers. ${ }^{3-9)}$ This approach has been applied to the numerical modeling for the damage progress with finite-element analysis. ${ }^{3)}$ Here after, we briefly introduce the previous works in this field.

Lee, Allen and Harris ${ }^{1)}$ derived approximate relationship between the internal state variable (ISV) and the stiffness of the laminate. They derived the upper limit of the effective Young' s modulus of the $\left[0_{q} / 90_{r}\right]_{s}$ laminate including transverse cracking in 
the $90^{\circ}$ ply by using the minimum potential energy principle.

Gudmundson and Zang ${ }^{2)}$ suggested a sophisticated model that predicts the thermoelastic properties of the laminate including transverse cracks. In this model, the average crack opening displacement of a transverse crack is assumed to be that of an isotropic elastic medium, and the incremental strain of the ply in which the transverse crack is initiated is calculated. They embedded this model into classical laminate theory, so that the relationship between transverse crack density of laminates with arbitrary lay-up configurations and their mechanical properties could be estimated analytically. Kobayashi et al. ${ }^{13)}$ derived the energy release rate for transverse cracks with this model and predicted the formation of transverse cracks in quasi-isotropic laminates. They deemed that the local stress distribution has not been determined, although the average stress distribution in each ply can be derived. In contrast, as discussed later, the model proposed in this paper can represent the local stress distribution in the ply including transverse cracks as a function of crack density.

For the quasi-isotropic $[0 / 90 /-45 /+45]_{s}$ laminate, Tong et al. ${ }^{14,15)}$ observed the damage evolution experimentally under quasi-static and fatigue loadings and investigated the mechanical properties of laminate using a FEM simulation that takes account 
of the experiment results. Based on the experiment results, when the laminate was subjected to quasi-static and fatigue loadings in the direction of $0^{\circ}$, cracks were observed in the $90^{\circ},-45^{\circ}$, and $+45^{\circ}$ plies. The crack size was smaller and the fatigue crack growth rate was slower in the $-45^{\circ}$ ply compared to that of the other plies.

The effective compliance of the laminate including transverse cracks was formulated using a damage variable of a second-rank symmetric tensor proposed by Murakami and his colleagues ${ }^{11,12)}$ in terms of continuum damage mechanics. They formulated the effective compliance of laminate as a function of the damage variable by embedding the effective compliance of the plies into classical laminate theory. However, they assumed that the damage variable is proportional to the crack density.

In this study, a model for predicting the stiffness reduction of composite laminates including transverse cracks is formulated as a function of crack density using twodimensional elasticity. In particular, the damage variable in the direction normal to the fiber was derived for the ply including transverse cracking. Here, the damage variable in the direction normal to the fiber was formulated from the model assuming a plane strain field in the isotropic plane and the Gudmundson-Zang ${ }^{2)}$ model. Using the effective compliance based on the strain equivalent principle formulated by Murakami et 
al. ${ }^{11,12)}$, the effective compliance of a ply was calculated with the damage variable stated above. Next, the effective compliance of a ply was embedded in classical laminate theory to formulate the effective compliance of laminates with arbitrary lay-up configurations as a function of crack density. Finally, the validity of this model was estimated by comparing the results obtained from this model to those in previous studies. ${ }^{2,14-17)}$

\section{Continuum damage mechanics model}

Damage mechanics model of composite laminates

It is difficult to directly derive a damage model for laminate because of the difference in damage formation in each ply in the laminate. Therefore, this paper investigated the mechanical behavior of plies damaged by transverse cracks. A damage model of the laminate was then formulated with the help of classical laminate theory. When formulating the damage model, the following assumptions were made: (i) the ply is thin, (ii) damage is mainly according to cracking due to transverse cracks. Based on these assumptions, a plane stress condition without considering the bending of the ply was assumed, and the damage due to delamination was ignored. In this section, based

on the study of Murakami et al. ${ }^{11,12)}$, the effective compliance of the damaged ply is 
shown, and the effective compliance of the laminate is derived.

First, the damage mechanics model of a ply is established. As seen in Fig. 1, the 1-axis is parallel to the fiber and the 2 and 3-axes are normal to the fiber. In the continuum damage mechanics model, the strain equivalent principle and the strain energy equivalent principle are known as a derivation method for determining the effective compliance. In this study, the effective compliance based on the strain equivalent principle was used. The strain equivalent principle is used as follows. The constitutive equation of damaged material under stress loading condition is written by substituting the effective stress for the stress term in the constitutive equation of undamaged material. By using the strain equivalent principle, the effective compliance of the ply damaged by transverse cracking seen in Fig. 1 is described as follows.

$$
C=C_{0} M
$$

Here, $\boldsymbol{C}_{0}$ is compliance under undamaged conditions and $\boldsymbol{M}$ is a damage effect tensor. In plane 1-2, the plane stress condition was assumed. When the in-plane stress and strain of a ply are defined as $\boldsymbol{\sigma}=\left[\sigma_{1}, \sigma_{2}, \sigma_{12}\right]^{T}$ and $\varepsilon=\left[\varepsilon_{1}, \varepsilon_{2}, 2 \varepsilon_{12}\right]^{T}, \boldsymbol{C}_{0}$ and $\boldsymbol{M}$ on 
the principal axis of the ply are specified as follows.

$$
\begin{aligned}
\boldsymbol{C}_{0}= & {\left[\begin{array}{ccc}
\frac{1}{E_{1}} & -\frac{\nu_{12}}{E_{1}} & 0 \\
-\frac{\nu_{12}}{E_{1}} & \frac{1}{E_{2}} & 0 \\
0 & 0 & \frac{1}{G_{12}}
\end{array}\right] } \\
\boldsymbol{M}= & {\left[\begin{array}{ccc}
1 & 0 & 0 \\
0 & \frac{1}{1-d_{2}} & 0 \\
0 & 0 & \frac{1}{2}\left(1+\frac{1}{1-d_{2}}\right)
\end{array}\right] }
\end{aligned}
$$

$E_{1}$ and $E_{2}$ are Young' s modulus along the 1 and 2-axes respectively, $G_{12}$ is a shear modulus in plane $1-2, \nu_{12}$ is Poisson' s ratio, and $d_{2}$ is the damage variable in the direction normal to the fiber. Damage variable $d_{2}$ is derived as a function of crack density $\rho$ in the following sections. Thus, if the damage due to the transverse crack is ignored, $d_{2}$ will be 0 .

Subsequently, the effective compliance of laminates of arbitrary lay-up configurations was derived based on the classical laminate theory. The coordinate system of the laminate is defined as $O-X Y Z$. Here, let us assume that the $X-Y$ plane is parallel to 
the 1-2 plane of the ply and the $Z$ axis is in the same direction as the 3 -axis. The effective compliance of the kth ply $(k=1,2, \cdots, N)$ is derived. The effective compliance of a ply $\boldsymbol{C}_{k}$ in the coordinate system of the principal axis of laminate $O-X Y Z$ is given in Eq. (1). The direction of a fiber tilted at a $\alpha_{k}$ degree angle to the $X$-axis is indicated in Fig. 2.

$$
\boldsymbol{C}_{k}=\boldsymbol{R}_{k} \boldsymbol{C} \boldsymbol{T}_{k}^{-1}=\boldsymbol{R}_{k} \boldsymbol{C}_{0} \boldsymbol{M} \boldsymbol{T}_{k}^{-1}
$$

Here, $\boldsymbol{R}_{k}$ and $\boldsymbol{T}_{k}$ are coordinate conversion matrices that are described as follows.

$$
\begin{aligned}
& \boldsymbol{R}_{k}=\left[\begin{array}{ccc}
\cos ^{2} \alpha_{k} & \sin ^{2} \alpha_{k} & -\sin \alpha_{k} \cos \alpha_{k} \\
\sin ^{2} \alpha_{k} & \cos ^{2} \alpha_{k} & \sin \alpha_{k} \cos \alpha_{k} \\
2 \sin \alpha_{k} \cos \alpha_{k} & -2 \sin \alpha_{k} \cos \alpha_{k} & \cos ^{2} \alpha_{k}-\sin ^{2} \alpha_{k}
\end{array}\right] \\
& \boldsymbol{T}_{k}=\left[\begin{array}{ccc}
\cos ^{2} \alpha_{k} & \sin ^{2} \alpha_{k} & -2 \sin \alpha_{k} \cos \alpha_{k} \\
\sin ^{2} \alpha_{k} & \cos ^{2} \alpha_{k} & 2 \sin \alpha_{k} \cos \alpha_{k} \\
\sin \alpha_{k} \cos \alpha_{k} & -\sin \alpha_{k} \cos \alpha_{k} & \cos ^{2} \alpha_{k}-\sin ^{2} \alpha_{k}
\end{array}\right]
\end{aligned}
$$


In classical laminate theory, the effective compliance of laminate $\bar{C}$ is described as

$$
\overline{\boldsymbol{C}}=\left(\frac{1}{t_{L}} \sum_{k=1}^{N} t_{k} \boldsymbol{C}_{k}^{-1}\right)^{-1}=\left(\frac{1}{t_{L}} \sum_{k=1}^{N} t_{k}\left(\boldsymbol{R}_{k} \boldsymbol{C}_{0} \boldsymbol{M} \boldsymbol{T}_{k}^{-1}\right)^{-1}\right)^{-1}
$$

where $t_{L}$ is the thickness of the laminate and $t_{k}$ is the thickness of the $k$-th ply. From Eq. (7), if the damage variable $d_{2}$, which is a component of the damage effect tensor $\boldsymbol{M}$, can be formulated, the mechanical properties of the damaged laminate can be obtained. In the following sections, the formulation of $d_{2}$ of a ply using the model assuming a plane strain field in the isotropic plane is conducted using the Gudmundson-Zang model.

Formulation of the damage variable in the direction normal to the fiber using the model assuming a plane strain field in the isotropic plane

This section derives the damage variable $d_{2}$ normal to the fiber by considering the damage due to transverse crack parallels to the fiber. Fig. 3 depicts a representative volume element including a transverse crack on both sides, which is a part of the ply seen in Fig. 1. The ply strain $\varepsilon_{y}^{p}$ is applied to the corresponding ply. $2 l$ is the crack distance and $2 t\left(=t_{k}\right)$ is the thickness of the ply. The transverse crack is assumed to have a 
tunnel-like crack surface which is symmetrical about the $y$ axis. The transverse crack does not propagate into the neighboring ply. It should be noted that the coordinate system differs from that in Fig. 1 in the previous section. The coordinates $x, y$, and $z$ in Fig. 3 correspond to the 3-, 2- and 1-axes in Fig. 1, respectively. Due to the symmetric model, the range is limited to $0 \leq x \leq t$ and $0 \leq y \leq l$. A plane strain condition in the isotropic plane ( $x-y$ plane) in Fig. 3 is assumed. The deformations in directions $x, y$, and $z$ are defined as $u, v$, and $w$. The stress-strain relation is expressed as follows.

$$
\begin{aligned}
\varepsilon_{x}(x, y) & =\frac{\partial u}{\partial x}(x, y)=C_{1} \sigma_{x}(x, y)-C_{2} \sigma_{y}(x, y) \\
\varepsilon_{y}(x, y) & =\frac{\partial v}{\partial y}(x, y)=C_{1} \sigma_{y}(x, y)-C_{2} \sigma_{x}(x, y) \\
\gamma_{x y} & =\frac{\sigma_{x y}(x, y)}{G_{23}}=\frac{\partial v}{\partial x}(x, y)+\frac{\partial u}{\partial y}(x, y) \approx \frac{\partial v}{\partial x}(x, y)
\end{aligned}
$$

The constants $C_{1}$ and $C_{2}$ are described as

$$
\begin{aligned}
& C_{1}=\frac{1-\nu_{12} \nu_{21}}{E_{2}}, \\
& C_{2}=\frac{\nu_{23}+\nu_{12} \nu_{21}}{E_{2}},
\end{aligned}
$$

where $\nu_{23}$ and $G_{23}$ are the Poisson' s ratio and shear modulus in the 2-3 plane in Fig. 
1. For the engineering shear strain $\gamma_{x y}$ in Eq. (10), the gradient in the $y$ direction of displacement $u$ is assumed to be quite small. The relationship between the strain in the $x$ direction $\varepsilon_{x}$ and the strain in the $y$ direction $\varepsilon_{y}$ is expressed as

$$
\varepsilon_{x}(x, y)=a \varepsilon_{y}(x, y),
$$

where $a$ is a proportionality constant defined to satisfy the equilibrium equation of stress. From Eqs. (8) and (9), Eq. (13) can be described as follows.

$$
a \frac{\partial v}{\partial y}(x, y)=C_{1} \sigma_{x}(x, y)-C_{2} \sigma_{y}(x, y)
$$

When $\sigma_{y}(x, y)$ in Eq. (9) is eliminated by using Eq. (14), $\sigma_{x}(x, y)$ is described as follows.

$$
\sigma_{x}(x, y)=\frac{a C_{1}+C_{2}}{C_{1}^{2}-C_{2}^{2}} \frac{\partial v}{\partial y}(x, y)
$$

In turn, when $\sigma_{x}(x, y)$ in Eq. (9) is eliminated using Eq. (14), $\sigma_{y}(x, y)$ is described as 
follows.

$$
\sigma_{y}(x, y)=\frac{C_{1}+a C_{2}}{C_{1}^{2}-C_{2}^{2}} \frac{\partial v}{\partial y}(x, y)
$$

Additionally, using Eq. (10), $\sigma_{x y}(x, y)$ is described as follows.

$$
\sigma_{x y}=G_{23} \frac{\partial v}{\partial x}(x, y)
$$

The equilibrium equation of stress is expressed as follows.

$$
\begin{gathered}
\frac{\partial \sigma_{x}}{\partial x}+\frac{\partial \sigma_{x y}}{\partial y}=0 \\
\frac{\sigma_{x y}}{\partial x}+\frac{\partial \sigma_{y}}{\partial y}=0
\end{gathered}
$$

When $\partial^{2} v / \partial x \partial y \neq 0$ is postulated and Eqs. (15) and (17) are substituted into Eq. (18), the proportionality constant $a$ is defined as follows.

$$
\begin{aligned}
a & =-\frac{C_{2}+G_{23}\left(C_{1}^{2}-C_{2}^{2}\right)}{C_{1}} \\
& =-\frac{E_{2}\left(\nu_{23}+\nu_{12} \nu_{21}\right)+G_{23}\left\{\left(1-\nu_{12} \nu_{21}\right)^{2}-\left(\nu_{23}+\nu_{12} \nu_{21}\right)^{2}\right\}}{E_{2}\left(1-\nu_{12} \nu_{21}\right)}
\end{aligned}
$$


By substituting Eqs. (16) and (17) for the stress term in Eq. (19), the Laplace equation for displacement $v$ is obtained as

$$
\frac{\partial^{2} v}{\partial x^{2}}+\lambda^{2} \frac{\partial^{2} v}{\partial y^{2}}=0
$$

where $\lambda$ is a constant value determined as follows.

$$
\lambda=\sqrt{\frac{C_{1}+a C_{2}}{G_{23}\left(C_{1}^{2}-C_{2}^{2}\right)}}=\sqrt{\frac{E_{2}-G_{23}\left(\nu_{23}+\nu_{12} \nu_{21}\right)}{G_{23}\left(1-\nu_{12} \nu_{21}\right)}}
$$

To determine displacement $v$, the boundary conditions of the Laplace equation in Eq. (21) are given as follows.

$$
\begin{aligned}
v(x, 0) & =0 \\
\frac{\partial v}{\partial y}(x, l) & =0 \\
\frac{\partial v}{\partial x}(0, y) & =0 \\
v(t, y) & =\varepsilon_{y}^{p} y
\end{aligned}
$$

At $y=0$, there is no displacement $v$ considered in Eq. (23). Equation (24) was 
determined from Eq. (16) considering the stress $\sigma_{y}=0$ at the crack surface $(y=l)$. Equation (25) was defined based on Eq. (17), considering no shear deformation $\sigma_{x y}=0$ on the center line of the ply $(x=0)$. Additionally, the displacement distribution shown as Eq. (26) in the interface was assumed according to the previous study. ${ }^{1)}$ Therefore, the neighboring ply is thought to be deformed uniformly by $\varepsilon_{y}^{p}$ regardless of the transverse cracks. Using separation of variables and assigning the boundary conditions shown above to Eq. (21), the solution $v(x, y)$ of the Laplace equation that satisfies the boundary conditions in Eqs. (23) to (26) can be described as follows.

$$
v(x, y)=\frac{8 l \varepsilon_{y}^{p}}{\pi^{2}} \sum_{n=1}^{\infty} \frac{(-1)^{n+1}}{(2 n-1)^{2}} \frac{\cosh \left(\frac{2 n-1}{2 l} \pi \lambda x\right)}{\cosh \left(\frac{2 n-1}{2 l} \pi \lambda t\right)} \sin \left(\frac{2 n-1}{2 l} \pi y\right)
$$

Damage variable $d_{2}$ in the direction normal to the fiber is represented by the proportion of the average ply strain $\varepsilon_{y}^{a}$, which ignores the crack opening displacement and the ply strain $\varepsilon_{y}^{p}$, which considers the crack opening displacement, $\varepsilon_{y}^{a} / \varepsilon_{y}^{p}$. Therefore, the proportion of average ply strain $\varepsilon_{y}^{a}$ and ply strain $\varepsilon_{y}^{p}$ is calculated using Eq. (27). At 
first, the average ply strain $\varepsilon_{y}^{a}$ was defined as follows.

$$
\varepsilon_{y}^{a}=\frac{1}{l t} \int_{0}^{t} v(x, l) d x
$$

By substituting Eq. (27) into Eq. (28), $\varepsilon_{y}^{a} / \varepsilon_{y}^{p}$ is derived as follows.

$$
\frac{\varepsilon_{y}^{a}}{\varepsilon_{y}^{p}}=\sum_{n=1}^{\infty} \frac{16}{(2 n-1)^{3} \pi^{3} \lambda t_{k}} \frac{\tanh \left(\frac{2 n-1}{2 l} \pi \lambda t_{k} \rho\right)}{\rho}
$$

where $\rho=1 /(2 l)$ is the crack density and the thickness of the ply was defined as $t_{k}=2 t$.

Finally, the damage variable $d_{2}$ in the direction normal to the fiber was formulated. Due to the plane stress condition in plane 1-2 in Fig. 1, the plane stress condition is also postulated in the $z-y$ plane. The relationship between the ply strain $\left[\varepsilon_{z}^{p}, \varepsilon_{y}^{p}, 2 \varepsilon_{z y}^{p}\right]^{T}$ and average ply stress $\left[\sigma_{z}^{a}, \sigma_{y}^{a}, \sigma_{z y}^{a}\right]^{T}$ in the $z-y$ plane is expressed as follows when using 
the global effective elastic compliance given in Eq. (1).

$$
\left[\begin{array}{c}
\varepsilon_{z}^{p} \\
\varepsilon_{y}^{p} \\
2 \varepsilon_{z y}^{p}
\end{array}\right]=\left[\begin{array}{ccc}
\frac{1}{E_{1}} & -\frac{\nu_{12}}{\left(1-d_{2}\right) E_{1}} & 0 \\
-\frac{\nu_{12}}{E_{1}} & \frac{1}{\left(1-d_{2}\right) E_{2}} & 0 \\
0 & 0 & \frac{1}{2 G_{12}}\left(1+\frac{1}{1-d_{2}}\right)
\end{array}\right]\left[\begin{array}{c}
\sigma_{z}^{a} \\
\sigma_{y}^{a} \\
\sigma_{z y}^{a}
\end{array}\right]
$$

Here, considering the loading state as $\left[\sigma_{z}^{a}, \sigma_{y}^{a}, \sigma_{z y}^{a}\right]^{T}=\left[0, \sigma_{y}, 0\right]^{T}$, the following relationship is obtained from Eq. (30).

$$
\varepsilon_{y}^{p}=\frac{\sigma_{y}}{\left(1-d_{2}\right) E_{2}}
$$

On the contrary, when $\sigma_{y}^{a}>>\nu_{23} \sigma_{x}^{a}$ is assumed, $\varepsilon_{y}^{a}$ is expressed as follows from Hooke' s law.

$$
\varepsilon_{y}^{a}=\frac{\sigma_{y}^{a}}{E_{2}}-\frac{\nu_{23}}{E_{2}} \sigma_{x}^{a} \approx \frac{\sigma_{y}^{a}}{E_{2}}
$$


Using Eq. (32), the damage variable $d_{2}$ is derived as follows from Eq. (31).

$$
d_{2}=1-\frac{\varepsilon_{y}^{a}}{\varepsilon_{y}^{p}}
$$

Substituting Eq. (29) into Eq. (33), damage variable $d_{2}$ in the direction normal to the fiber is described as a function of the crack density $\rho$ as follows.

$$
d_{2}=1-\sum_{n=1}^{\infty} \frac{16}{(2 n-1)^{3} \pi^{3} \lambda t_{k}} \frac{\tanh \left(\frac{2 n-1}{2} \pi \lambda t_{k} \rho\right)}{\rho}
$$

In the equation above, $t_{k}$ is the thickness of the ply in the laminate, and $\lambda$ is the constant derived from the elastic moduli of a ply in Eq. (22). The damage effect tensor $\boldsymbol{M}$ seen in Fig. 3 can be calculated using Eq. (34). The change in the elastic moduli of the laminate due to a change in the transverse crack density is obtained by determining the elastic moduli of a ply and the laminated constitution. According to classical laminate theory, this method can apply to laminates with arbitrary lay-up configurations.

As seen in Fig. 4 (a), a crack may occur in the laminate surface. In thin laminated, cracks in the surface seriously affect the mechanical properties. Fig. 4 (b) presents a 
representative volume element of a ply that has a crack in the surface. In this case, the area of $0 \leq x \leq 2 t$ and $0 \leq y \leq l$ should be considered as pointed out by Gudmundson and Zang. The surface crack can be considered by replacing the thickness of a ply $t_{k}$ with $2 t_{k}$ in Fig. 3. By replacing $t_{k}$ with $2 t_{k}$, in Eq. (34), the damage variable $d_{2}$ in the direction normal to the fiber considering the surface crack may be represented as follows.

$$
d_{2}=1-\sum_{n=1}^{\infty} \frac{8}{(2 n-1)^{3} \pi^{3} \lambda t_{k}} \frac{\tanh \left\{(2 n-1) \pi \lambda t_{k} \rho\right\}}{\rho}
$$

When transverse cracks in a ply is considered, Eq. (34) is appropriate, as Eq. (35) is appropriate for a surface crack. When Eq. (27) is substituted into Eqs. (15) to (17), the stress at $P(x, y)$ is derived as follows.

$$
\begin{aligned}
\sigma_{x}(x, y) & =\frac{4}{\pi} \frac{a C_{1}+C_{2}}{C_{1}^{2}-C_{2}^{2}} \varepsilon_{y}^{p} \sum_{n=1}^{\infty} \frac{(-1)^{n+1}}{2 n-1} \frac{\cosh \left(\frac{2 n-1}{2 l} \pi \lambda x\right)}{\cosh \left(\frac{2 n-1}{2 l} \pi \lambda t\right)} \cos \left(\frac{2 n-1}{2 l} \pi y\right) \\
\sigma_{y}(x, y) & =\frac{4}{\pi} \frac{C_{1}+a C_{2}}{C_{1}^{2}-C_{2}^{2}} \varepsilon_{y}^{p} \sum_{n=1}^{\infty} \frac{(-1)^{n+1}}{2 n-1} \frac{\cosh \left(\frac{2 n-1}{2 l} \pi \lambda x\right)}{\cosh \left(\frac{2 n-1}{2 l} \pi \lambda t\right)} \cos \left(\frac{2 n-1}{2 l} \pi y\right) \\
\sigma_{x y}(x, y) & =\frac{4 \lambda G_{23}}{\pi} \varepsilon_{y}^{p} \sum_{n=1}^{\infty} \frac{(-1)^{n+1}}{2 n-1} \frac{\sinh \left(\frac{2 n-1}{2 l} \pi \lambda x\right)}{\cosh \left(\frac{2 n-1}{2 l} \pi \lambda t\right)} \sin \left(\frac{2 n-1}{2 l} \pi y\right)
\end{aligned}
$$


The stress distribution in a ply including transverse cracks is obtained from Eqs. (36) to (38). As seen in Eqs. (36) to (38), the local stress distribution in the ply including transverse cracks can be calculated by using our model. In contrast, only the average stress in each ply in the laminate can be calculated by the Gudmundson-Zang model. This is the difference from the Gudmundson-Zang model.

Formulation of damage variables in the direction normal to the fiber using the GudmundsonZang model

As seen in Eq. (33), damage variable $d_{2}$ can be represented by the portion of $\varepsilon_{y}^{a} / \varepsilon_{y}^{p}$. Therefore, damage variable $d_{2}$ can be represented in various manners by deriving $\varepsilon_{y}^{a} / \varepsilon_{y}^{p}$ in some way. Here, by applying the Gudmundson-Zang model, which can predict the thermo-elastic properties of a laminate including transverse cracks, the damage variable $d_{2}$ was formulated.

According to the Gudmundson-Zang model, the increment of the in-plane strain due to the crack opening in the $z-y$ plain in Fig. 3 can be represented as

$$
\Delta \varepsilon_{I}=t_{k} \rho\left(\boldsymbol{N}_{I}\right)^{T} \boldsymbol{\beta} \boldsymbol{N}_{I}\left(\boldsymbol{C}_{0}\right)^{-1} \varepsilon_{I}^{p}
$$


where $t_{k}$ is the thickness of the ply, $\rho$ is the crack density in the corresponding ply, $\varepsilon_{I}^{p}=\left[\varepsilon_{z}^{p}, \varepsilon_{y}^{p}, 2 \varepsilon_{z y}^{p}\right]^{T}$ is the ply strain in the $z-y$ plane, and $\boldsymbol{C}_{0}$ is the compliance of the undamaged ply derived from Eq. (2). Additionally, $\boldsymbol{N}_{I}$ is a matrix determined from the normal unit vector defined as $\boldsymbol{n}=\left[n_{x}, n_{y}, n_{z}\right]^{T}$ that is perpendicular to the transverse crack in the ply.

$$
\boldsymbol{N}_{I}=\left[\begin{array}{ccc}
n_{z} & 0 & n_{y} \\
0 & n_{y} & n_{z} \\
0 & 0 & 0
\end{array}\right]=\left[\begin{array}{lll}
0 & 0 & 1 \\
0 & 1 & 0 \\
0 & 0 & 0
\end{array}\right] \text {, }
$$

where a normal unit vector is defined as $\boldsymbol{n}=[0,1,0]^{T}$, as seen in Fig. 3. Moreover, $\boldsymbol{\beta}$ is a matrix that connects the average crack opening displacement due to the transverse crack with the vector of the surface force affecting the surface of the transverse crack, which can be represented as follows.

$$
\boldsymbol{\beta}=\left[\begin{array}{ccc}
\beta_{1} & 0 & 0 \\
0 & \beta_{2} & 0 \\
0 & 0 & \beta_{3}
\end{array}\right]
$$


The ply strain $\varepsilon_{I}^{p}$ in the $z-y$ plane is represented as the sum of the average ply strain $\varepsilon_{I}^{a}=\left[\varepsilon_{z}^{a}, \varepsilon_{y}^{a}, \varepsilon_{z y}^{a}\right]$ and the increment of the ply strain $\Delta \varepsilon_{I}$.

$$
\varepsilon_{I}^{p}=\varepsilon_{I}^{a}+\Delta \varepsilon_{I}
$$

When Eq. (39) is substituted into Eq. (42), the following equation is derived.

$$
\varepsilon_{I}^{a}=\left(\boldsymbol{I}-t_{k} \rho\left(\boldsymbol{N}_{I}\right)^{T} \boldsymbol{\beta} \boldsymbol{N}_{I}\left(\boldsymbol{C}_{0}\right)^{-1}\right) \boldsymbol{\varepsilon}_{I}^{p},
$$

where $\boldsymbol{I}$ is the identity matrix. Here, we consider the following strain state $\varepsilon_{I}^{p}$ given by

$$
\varepsilon_{I}^{p}=\left[0, \varepsilon_{y}^{p}, 0\right]^{T}
$$

After Eqs. (2), (40), (41), and (44) are substituted into Eq. (43) and the components related to the second line are calculated, $\varepsilon_{y}^{a} / \varepsilon_{y}^{p}$ is derived as follows.

$$
\frac{\varepsilon_{y}^{a}}{\varepsilon_{y}^{p}}=1-\frac{E_{1} E_{2} t_{k}}{E_{1}-E_{2} \nu_{12}^{2}} \beta_{2} \rho
$$


By substituting Eq. (45) for Eq. (33), damage variable $d_{2}$ in the direction normal to the fiber is represented as follows.

$$
d_{2}=\frac{E_{1} E_{2} t_{k}}{E_{1}-E_{2} \nu_{12}^{2}} \beta_{2} \rho
$$

In the equation above, $\beta_{2}$ is a function of the crack density; for a transverse crack initiated in the ply it is represented as follows.

$$
\beta_{2}=\frac{\pi}{2} \frac{1-\nu_{12} \nu_{21}}{E_{2}} \sum_{j=1}^{10} \frac{a_{j}}{\left(1+t_{k} \rho\right)^{j}}
$$

When surface cracks are considered, $\beta_{2}$ is given as

$$
\beta_{2}=2(1.12)^{2}\left(\frac{\pi}{2} \frac{1-\nu_{12} \nu_{21}}{E_{2}} \sum_{j=1}^{10} \frac{c_{j}}{\left(1+t_{k} \rho\right)^{j}}\right)
$$

where the constant sequence $a_{j}$ and $c_{j}$ is given in Table 1. As seen in Eq. (46), damage variable $d_{2}$ in the direction normal to the fiber can be represented as a function of the crack density $\rho$ when the thickness of the ply $t_{k}$ and the elastic modulus of the ply are given. The elastic moduli of the laminates of arbitrary lay-up configurations 
that are changed by the transverse crack density are derived from Eq. (7) by giving the elastic modulus of the ply and the laminated constitution as discusses in previous section. However, as stated above and pointed out by Kobayashi ${ }^{13)}$, the GudmundsonZang model does not give stress and strain fields around the transverse crack.

\section{Results and discussion}

By using the two models formulated in this paper (the model assuming a plane strain field in the isotropic plane and the Gudmundson-Zang model), the elastic moduli of the laminate were calculated as a function of crack density to compare with the experiment $^{2,14-17)}$ and with the finite-element analytical results in previous works on GFRP and CFRP laminates. ${ }^{2}$ The validity of this model was evaluated for crossply, angle-ply, and quasi-isotropic laminates. When considering the damage due to transverse cracks in the laminate, damage variable $d_{2}$ was calculated using Eq. (34) or Eqs. (46) and (47). In the case of considering surface cracks, damage variable $d_{2}$ was calculated using Eq. (35) or Eqs. (46) and (48). The mechanical properties of the ply of GFRP and CFRP used in the calculation of the elastic moduli of laminate are listed in Table 2. Indexes 1, 2, and 3 in Table 2 represent the principal axes of the ply defined 
in Fig. 1. Additionally, it is assumed that the thickness of the plies $t_{k}$ is assigned as $t_{0}$ listed in Table 2 for all plies.

\section{Cross-ply laminate}

Here, the elastic modulus of cross-ply laminate is discussed. To calculate elastic moduli, the damage due to transverse cracks in the $90^{\circ}$ ply was considered.

First, a cross-ply $[90 / 0]_{s}$ GFRP laminate with a surface crack in the $90^{\circ}$ plies was considered. A representative periodic cell of the laminate is depicted in Fig. 5 (a). The material properties of GFRP-1 listed in Table 2 were used for the calculation. Figures 5 (b) and (c) give comparisons of the results for Young' s moduli and Poisson' s ratio for the laminate obtained from the Gudmundson-Zang model and the present model assuming a plane strain field in the isotropic plane, respectively. $E_{L 1}$ is the elastic modulus in the $X$ direction of the principal axis of the laminate, $\nu_{L 12}$ is the Poisson's ratio in the $X-Y$ plane of the laminate, and $\rho_{N}$ is the normalized crack density, which is the product of the thickness of each ply $t_{0}$ and the crack density $\rho$. Based on Figs. 5 (b) and (c), the results of the two models agree well with the analytical results obtained from the finite-element method. From this result, the elastic modulus and Poisson's 
ratio using damage variable $d_{2}$ derived from Eqs. (46) and (48) are lower than the values obtained from Eq. (35). Moreover, greater effects of the damage are obtained from the Gudmundson-Zang model than from the present model assuming a plane strain field in the isotropic plane.

The relationship between the normalized elastic moduli and normalized crack density of $[0 / 90]_{s}$ and $\left[0_{2} / 90_{2}\right]_{s}$ CFRP laminates with transverse cracks in the $90^{\circ}$ plies is illustrated in Fig. 6. $E_{L 10}$ is the elastic modulus in the $X$ direction of the undamaged laminate. The experiment results obtained by Groves et al. ${ }^{16)}$ are also shown in Fig. 6. From Fig. 6, the results obtained from the two models shows good agreement with the results obtained by Groves et al. Moreover, the results obtained from the present model assuming a plane strain field in the isotropic plane shows better agreement than that of the Gudmundson-Zang model.

The relationship between normalized elastic moduli and normalized crack density of $\left[0 / 90_{3}\right]_{s}$ GFRP laminate with transverse cracks in the $90^{\circ}$ plies is illustrated in Fig. 7. The material properties of GFRP-1 listed in Table 2 are used in the calculation. The experiment results of Highsmith and Reifsnider et al. ${ }^{17)}$ also appear in Fig. 7. From Fig. 7, as the crack density increases, the difference between the results obtained 
from the calculation using two models and those from the experiment increases. The reason for the difference between the results obtained from calculation and experiment involves damage formation such as local delamination and damage in $0^{\circ}$ plies. For Fig. 7, the result obtained from the Gudmundson-Zang model that show a greater effect of damage is in good agreement with the experiment results.

The normalized elastic moduli and normalized crack density as a function of crack density of $[0 / 90]_{s}$ GFRP laminate including transverse cracks in the $90^{\circ}$ plies are presented in Figs. 8 (a) and (b), respectively. $\nu_{L 120}$ is Poisson' s ratio in $X-Y$ plane of an undamaged laminate. The material properties of GFRP-2 listed in Table 2 are used for the calculation. Figs. 8 (a) and (b) present the experiment results of Tong et al. ${ }^{14)}$ Based on Figs. 8 (a) and (b), the result calculated from the present model assuming a plane strain field in the isotropic plane is in good agreement with the experiment results in the region under $0.3 \mathrm{~mm}^{-1}$ of crack density, but the experiment results dramatically decrease above $0.3 \mathrm{~mm}^{-1}$. The difference between the results obtained from calculation and from the experiment is due to damage formation such as local delamination and damage in the $0^{\circ}$ plies. 
Angle-ply laminate

This section discusses angle-ply GFRP laminate exhibiting damage due to transverse cracks in each ply. The analysis was conducted relative to $[ \pm 55]$ and $[ \pm 67.5]$ angleply laminate. The material properties of GFRP-1 listed in Table 2 are used in the calculation. The calculation was conducted with the elastic modulus, shear moduli and Poisson' s ratio as a function of normalized crack density. The analytical results for the $[ \pm 55]$ laminate are presented in Figs. 9 (a)-(c) and that for the $[ \pm 67.5]$ laminate is presented in Figs. 10 (a)-(c). $E_{L 1}$ and $E_{L 2}$ are the elastic moduli of the laminate in the $X$ and $Y$ directions of the principal axis of the laminate, $G_{L 12}$ is the shear modulus in the $X-Y$ plane and $\nu_{12}$ is Poisson' s ratio in the $X-Y$ plane. Figs. 9 (a)-(c) and 10 (a)-(c) also provide the analytical results for the Gudmundson-Zang model. Based on Figs. 9 and 10, the results obtained from these analytical models are in good agreement with the FEA result of the Gudmundson-Zang model, although that obtained from the Gudmundson-Zang model underestimate the elastic moduli compared to that obtained from the present model assuming a plane strain field in the isotropic plane. 
Quasi-isotropic laminate

This section analyzes the quasi-isotropic $[0 / 90 /-45 /+45]_{s}$ laminate and compares it to experiment results obtained by Tong et al. ${ }^{14,15)}$ The material used is GFRP and the material properties of GFRP-2 listed in Table 2 are used. The calculation was conducted assuming approximately the same damage occurrence due to transverse cracking in the $90^{\circ}$ and $\pm 45^{\circ}$ plies. The normalized elastic moduli and normalized Poisson' s ratio obtained from the calculation using the model assuming a plane strain field in the isotropic plane and the Gudmundson-Zang model are presented in Figs. 11 (a) and (b) respectively. Based on Figs. 11 (a), the results obtained from the present model are in good agreement with the experiment results, although those obtained from the Gudmundson-Zang model underestimate the elastic moduli. In Fig. 11(b), the results obtained from the present model show the deviations at high crack densities that were attributed to delamination and damage in 0 plies.

The model assuming a plane strain field in the isotropic plane proposed in this paper can calculate the local stress distribution in a ply including transverse cracking as a function of crack density. Therefore, the damage evolution of the transverse crack can be simulated by determining the fracture criterion. Such a simulation based on the 
present model is our future task.

\section{Conclusions}

In this paper, the damage variable is formulated by a model assuming a plane strain field in the isotropic plane and the Gudmundson-Zang model. The effective compliance based on the strain equivalent principle proposed by Murakami et al. and classical laminate theory is used to formulate the elastic moduli of laminates of arbitrary layup configurations as a function of crack density. The model proposed in this paper considers the effect of damage due to transverse cracks (or surface cracks) based only on the mechanical properties of the ply and the laminate constitution. Furthermore, this model can precisely predict the FEA results and experiment results for the elastic moduli of a laminate of arbitrary lay-up configuration, such as cross-ply, angle ply, and quasi-isotropic, including transverse cracks. Since this model only considers the damage from transverse cracks, it is impossible to consider the effect of other damage such as delamination. However, this model seems to be effective in the early stage of the damage formation during which transverse crack mainly occurs. 


\section{Acknowledgements}

T. O. thanks to the support of the Cross-ministerial Strategic Innovation Promotion Program and the Ministry of Education, Culture, Sports, Science and Technology of Japan under Grant-in-Aid Scientic Research (C) No. 15K06597. This work was partly supported by Toray Industries, Inc. This work was also partly supported by the New Energy and Industrial Technology Development Organization (NEDO) No. 151023150, P15006, and 141001611-d.

\section{References}

1) Lee JW, Allen DH and Harris CE. Internal state variable approach for predicting stiffness reductions in fibrous laminated composites with matrix cracks, J. Comp. Mater. 1989; 23(12): 1273-1291.

2) Gudmundson P and Zang W. An analytic model for thermoelastic properties of composite laminates containing transverse matrix cracks, Int. J. Solids Structures 1993; 30(23): 3211-3231. 
3) Tay TE and Lim EH. Analysis of composite laminates with transverse cracks, Composite Structures 1996; 34: 419-426.

4) Vyas GM and Pinho ST. Computational implementation of a novel constitutive model for multidirectional composites, Computational Materials Science 2012; 51: $217-224$.

5) Talreja R. A Continuum Mechanics Characterization of Damage in Composite Materials, Proc. R. Soc. Lond. 1985; 399 A.

6) Talreja R. Transverse Cracking and Stiffness Reduction in Composite Laminates, J. of Composite Materials 1985; 19: 355-375.

7) Talreja R. Stiffness Properties of Composite Laminates with Matrix Cracking and Interior Delamination, Engineering Fracture Mechanics 1986; 25 (5/6): 751-762.

8) Allen DH, Harris CE and Groves SE. A Thermomechanical Constitutive Theory for Elastic Composites with Distributed Damage - Part I: Theoretical Development, Int. J. of Solids and Structures 1987; 23(9): 1301-1318. 
9) Allen DH, Harris CE and Groves SE. A Thermomechanical Constitutive Theory for Elastic Composites with Distributed Damage - Part II: Application to Matrix Cracking in Laminated Composites Int. J. of Solids and Structures 1987; 23(9): 1319-1338.

10) Ogihara S, Takeda N, Kobayashi S and Kobayashi A. Damage mechanics analysis of transverse cracks in composite laminates, J. Soc. Mat. Sci., Japan 1998; 47(1): 68-72.

11) Murakami S. Continuum Damage Mechanics: A Continuum Mechanics Approach to the Analysis of Damage and Fracture. Dordrecht Heidelberg London New York: Springer, 2012.

12) Kanagawa Y, Murakami S, Liu Y, Bai Q and Tanaka K. Description of Internal Damage in FRP Laminates by Continuum Damage Mechanics, J. Soc. Mat. Sci., Japan 1996; 45(2): 206-211.

13) Kobayashi S, Ogihara S and Takeda N. Damage mechanics analysis for predicting mechanical behavior of general composite laminates containing transverse cracks, Adv. Composite Mater. 2000; 9(4): 363-375. 
14) Tong J, Guild FJ, Ogin SL and Smith PA. On matrix crack growth in quasi-isotropic laminates - I. experimental investigation, Comp. Sci. Tech. 1997; 57: 1527-1535.

15) Tong J, Guild FJ, Ogin SL and Smith PA. On matrix crack growth in quasi-isotropic laminates - II. finite element analysis, Comp. Sci. Tech. 1997; 57: 1537-1545.

16) Groves SE, Harris CE, Highsmith AL, Allen DH and Norvell RG. An experimental and analytical treatment of matrix cracking in cross-ply laminates, Exper. Mech. 1987; 27(1): 73-79.

17) Highsmith AL and Reifsnider KL. Stiffness reduction mechanism in composite laminates. In: Reifsnider KL (ed) Damage in Composite Materials, ASTM SPT 775, American Society for Testing and Materials, 1982, pp. 103-117.

18) Xia ZC, Carr RR and Hutchinson JW. Transverse cracking in fiber-reinforced brittle matrix, cross-ply laminates, Acta Metall. Mater. 1993; 41(8): 2365-2376.

19) Berthelot JM, Leblond P, El Mahi A and Le Corre JF. Transverse cracking of cross-ply laminates: Part 1. Analysis, Composites Part A 1996; 27(10): 989-1001. 
20) Berthelot JM, Le Corre JF. Statistical analysis of the progression of transverse cracking and delamination in cross-ply laminates, Composites Science and Technology 2000; 60(14): 2659-2669.

21) Berthelot JM. Transverse cracking and delamination in cross-ply glass-fiber and carbon-fiber reinforced plastic laminates: Static and fatigue loading, Applied Mechanics Reviews 2003; 56(1): 111-147.

22) Hashin Z. Analysis of stiffness reduction of cracked cross-ply laminates, Engineering Fracture Mechanics 1986; 25(5-6):771-778.

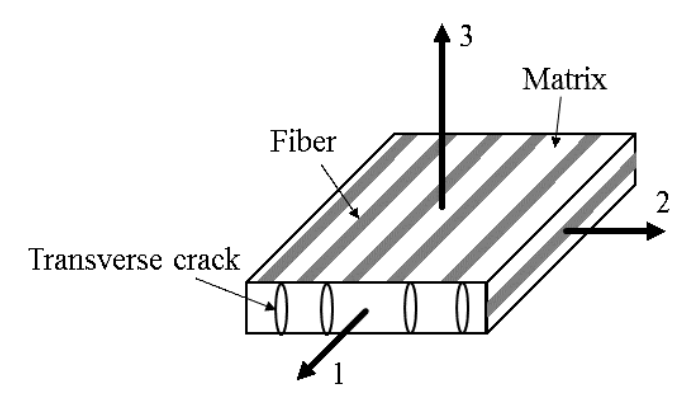

Figure 1 Ply including transverse crack 


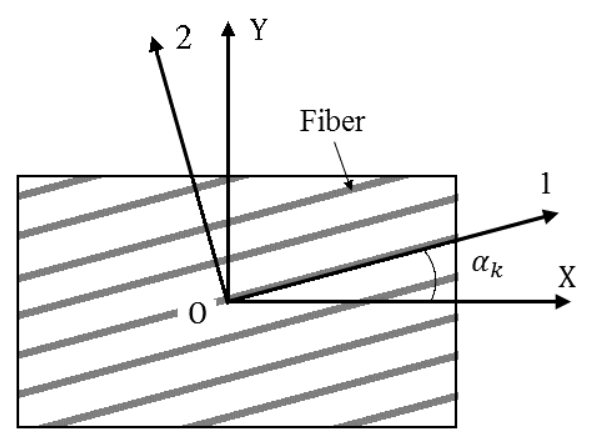

Figure 2 In-plane coordinate conversion

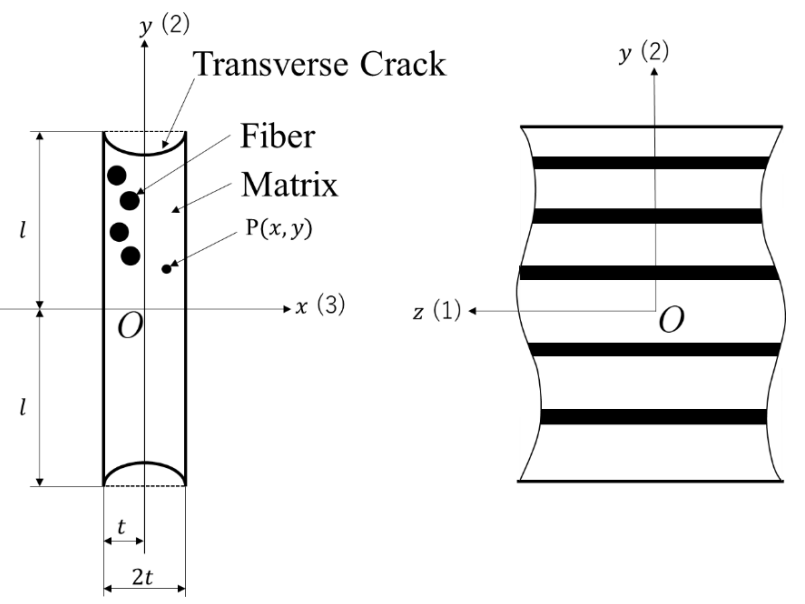

Figure 3 Transverse crack model 


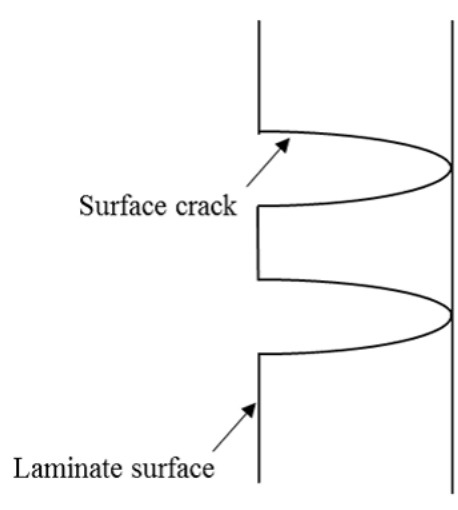

(a) Surface crack

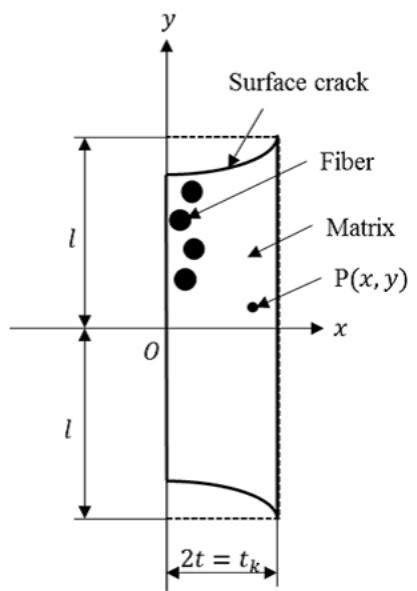

(b) Representative Volume Element

Figure 4 Surface crack model

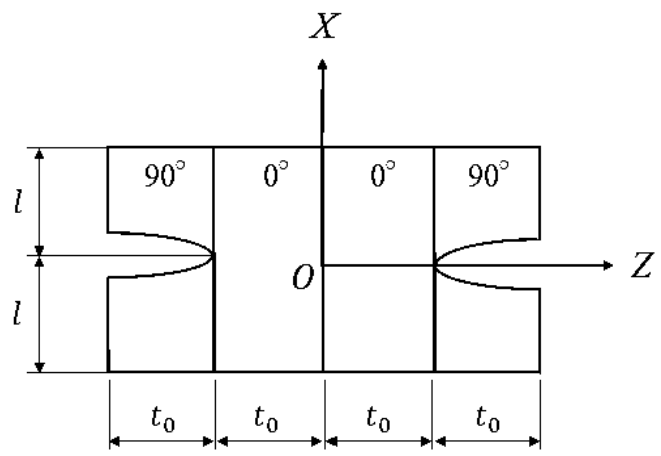

Figure 5 (a) Representative periodic cell for a cross-ply laminate with surface cracks. 


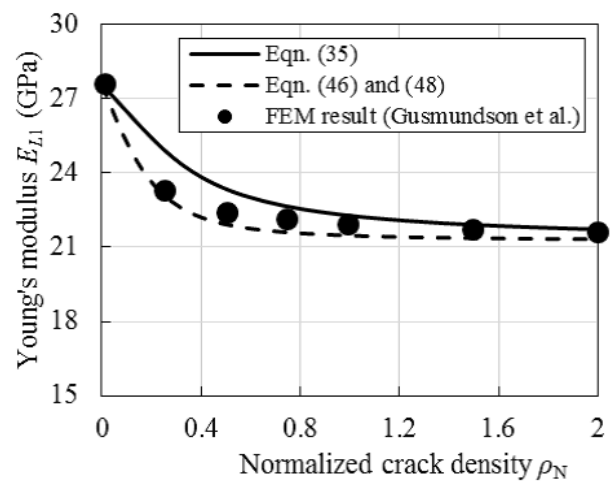

Figure 5 (b) Young's modulus as a function of normalized crack density in the $90^{\circ}$ plies for $[90 / 0]_{s}$ GFRP laminate with surface cracks.

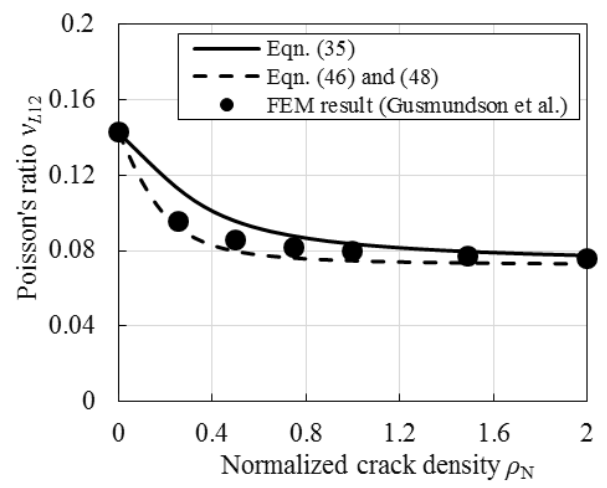

Figure 5 (c) Poisson's s ratio as a function of normalized crack density in the $90^{\circ}$ plies for $[90 / 0]_{s}$ GFRP laminate with surface cracks. 


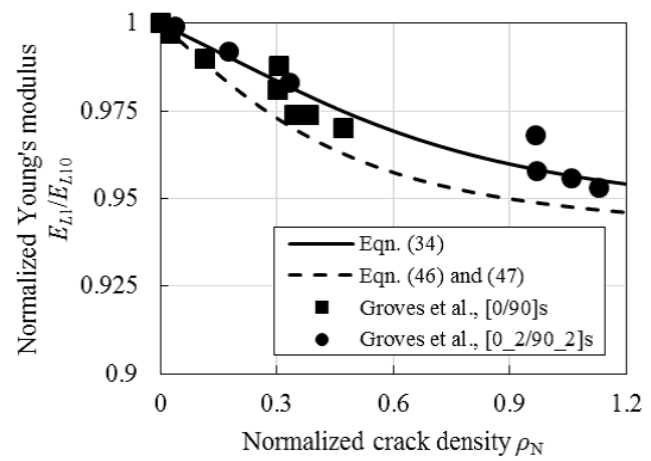

Figure 6 Normalized Young's modulus as a function of normalized crack density in the $90^{\circ}$ plies for $[0 / 90]_{s}$ and $\left[0_{2} / 90_{2}\right]_{s}$ CFRP laminate.

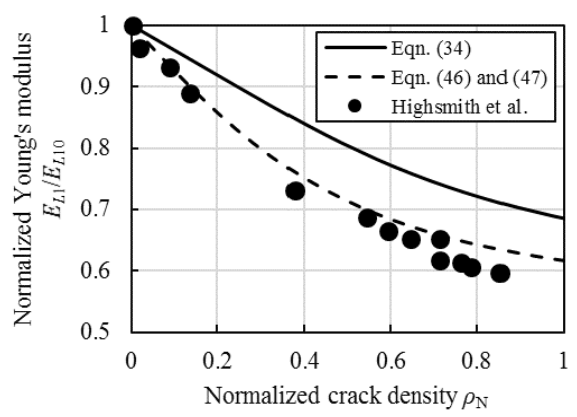

Figure 7 Normalized Young's modulus as a function of normalized crack density in the $90^{\circ}$ plies for $\left[0 / 90_{3}\right]_{s}$ GFRP laminate. 


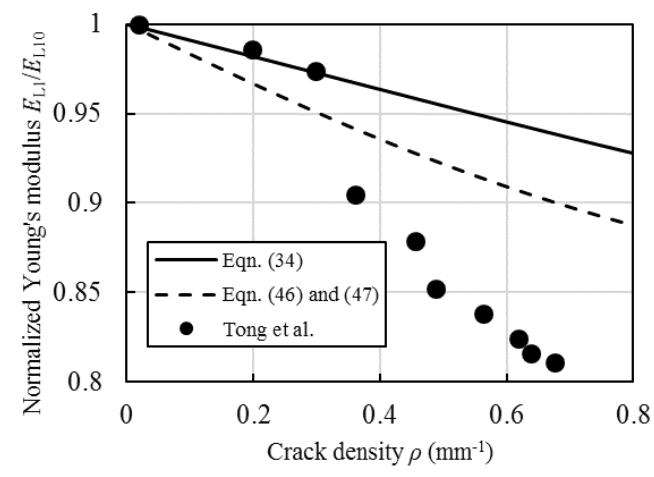

Figure 8 (a) Normalized Young' s modulus as a function of crack density in the $90^{\circ}$ plies for $[0 / 90]_{s}$ GFRP laminate.

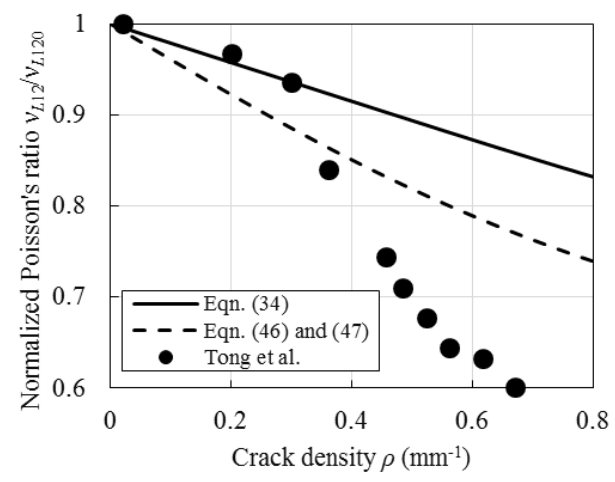

Figure 8 (b) Normalized Poisson' s ratio as a function of crack density in the $90^{\circ}$ plies for $[0 / 90]_{s}$ GFRP laminate. 


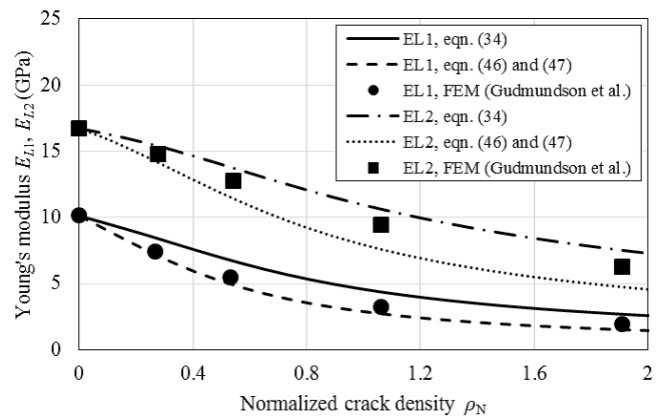

Figure 9 (a) Young's modulus as a function of normalized crack density for $[ \pm 55]$ GFRP laminate.

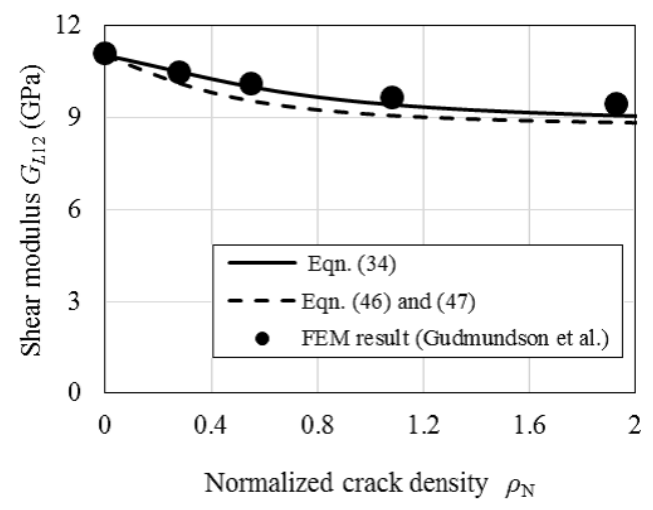

Figure 9 (b) Shear modulus as a function of normalized crack density for [ \pm 55$]$ GFRP laminate. 


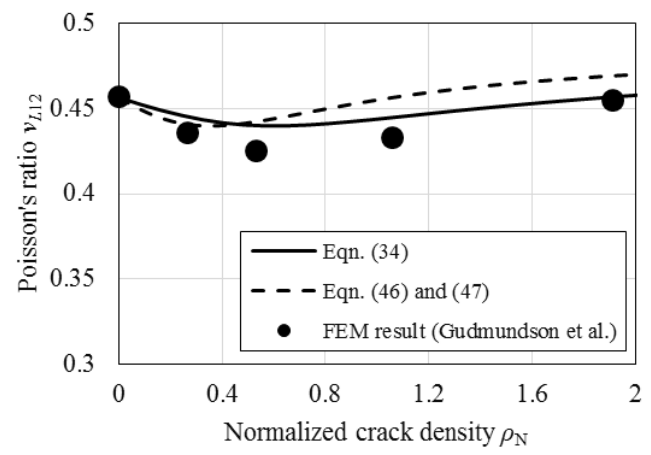

Figure 9 (c) Poisson's ratio as a function of normalized crack density for [ \pm 55$]$ GFRP laminate.

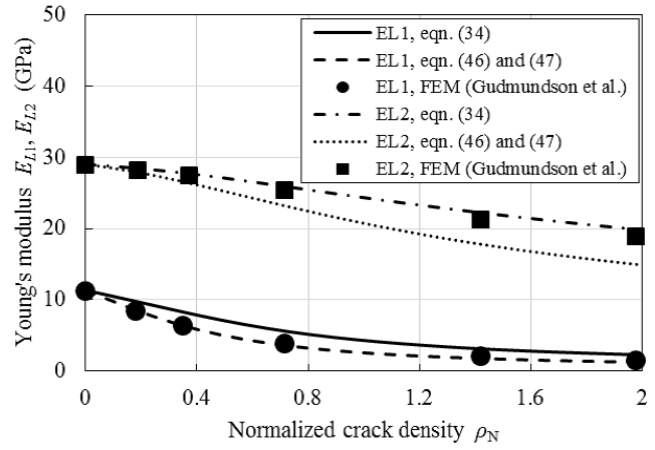

Figure 10 (a) Young' s modulus as a function of normalized crack density for $[ \pm 67.5]$ GFRP laminate. 


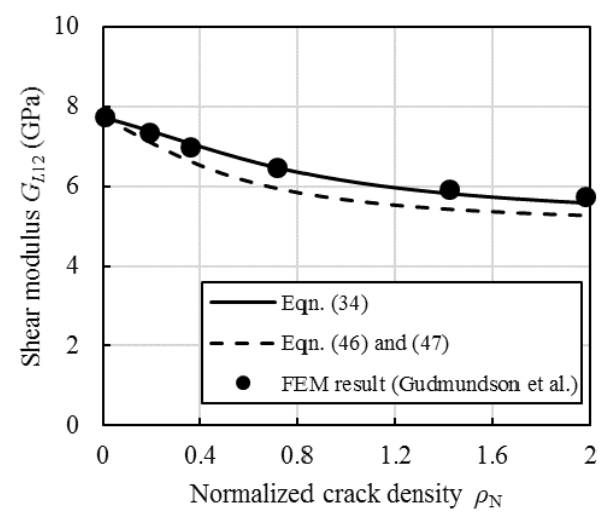

Figure 10 (b) Shear modulus as a function of normalized crack density for $[ \pm 67.5]$ GFRP laminate.

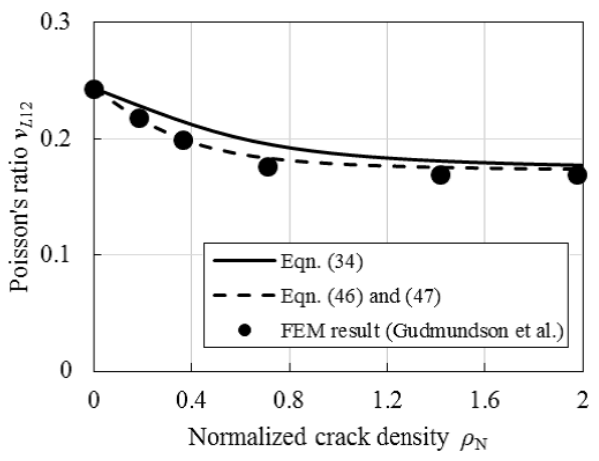

Figure 10 (c) Poisson's ratio as a function of normalized crack density for $[ \pm 67.5]$ GFRP laminate. 


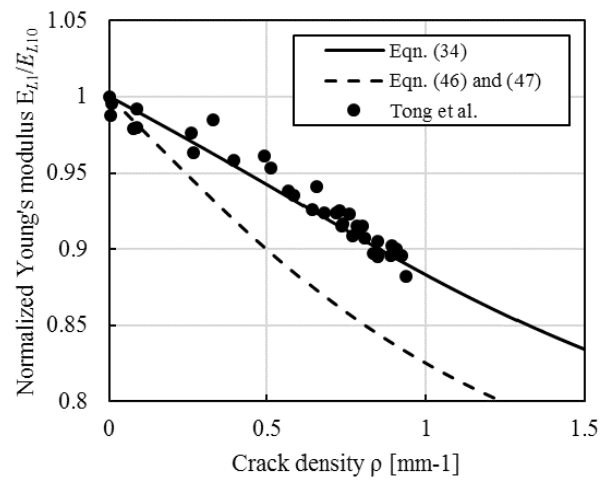

Figure 11 (a) Normalized Young' s modulus as a function of crack density for [0/90/ $45 /+45]_{s}$ GFRP laminate. 


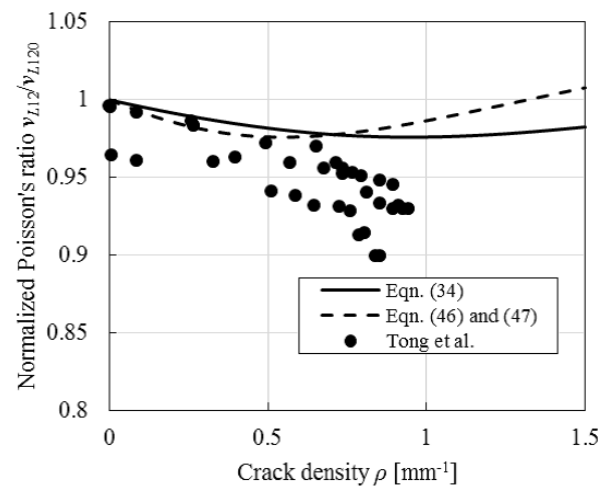

Figure 11 (b) Normalized Poisson's s ratio as a function of crack density for [0/90/ $45 /+45]_{s}$ GFRP laminate. 
Table 1 Numerical parameters used in Eqs. (47) and $(48)^{2)}$.

\begin{tabular}{c|c|c}
\hline$j$ & $a_{j}$ & $c_{j}$ \\
\hline 1 & 0.63666 & 0.25256 \\
\hline 2 & 0.51806 & 0.27079 \\
\hline 3 & 0.51695 & -0.49814 \\
\hline 4 & -1.04897 & 8.62962 \\
\hline 5 & 8.95572 & -51.24655 \\
\hline 6 & -33.09444 & 180.96305 \\
\hline 7 & 74.32002 & -374.29813 \\
\hline 8 & -103.06411 & 449.59474 \\
\hline 9 & 73.60337 & -286.51016 \\
\hline 10 & -20.34326 & 73.84223 \\
\hline
\end{tabular}


Table 2 Material properties for GFRP and CFRP unidirectional plies.

\begin{tabular}{c|c|c|c|c|c|c|c}
\hline Type & $E_{1}$ & $E_{2}$ & $\nu_{12}$ & $\nu_{23}$ & $G_{12}$ & $G_{23}$ & Ply thickness \\
& $(\mathrm{GPa})$ & $(\mathrm{GPa})$ & & $(\mathrm{GPa})$ & $(\mathrm{GPa})$ & $t_{0}(\mathrm{~mm})$ \\
\hline GFRP-1 & 41.7 & 13 & 0.3 & 0.42 & 3.4 & 4.58 & 0.203 \\
\hline GFRP-2 & 46 & 13 & 0.3 & 0.42 & 5 & 4.6 & 0.5 \\
\hline CFRP & 142 & 9.85 & 0.3 & 0.46 & 4.48 & 3.37 & 0.127 \\
\hline
\end{tabular}

